\title{
Activation of Astrocytes by CNTF Induces Metabolic Plasticity and Increases Resistance to Metabolic Insults
}

\author{
Carole Escartin, ${ }^{1,2,3}$ Karin Pierre, ${ }^{3}$ Angélique Colin, ${ }^{1,2}$ Emmanuel Brouillet, ${ }^{1,2}$ Thierry Delzescaux, ${ }^{2}$ \\ Martine Guillermier, ${ }^{1,2}$ Marc Dhenain, ${ }^{1,2}$ Nicole Déglon, ${ }^{1,2}$ Philippe Hantraye, ${ }^{1,2}$ Luc Pellerin, ${ }^{3}$ and Gilles Bonvento ${ }^{1,2}$ \\ ${ }^{1}$ Commissariat à l'Energie Atomique-Direction des Sciences du Vivant, Institut d'Imagerie Biomédicale, Service Hospitalier Frederic Joliot, Centre National \\ de la Recherche Scientifique, Unité de Recherche Associée 2210, 91401 Orsay, France, ${ }^{2}$ Commissariat à l'Energie Atomique-Direction des Sciences du \\ Vivant, Institut d’Imagerie Biomédicale, Molecular Imaging Research Center, 92265 Fontenay-aux-roses, France, and ${ }^{3}$ Département de Physiologie, \\ Université de Lausanne, Lausanne 1005, Switzerland
}

High energy demands of neurons make them vulnerable to adverse effects of energy impairment. Recently, astrocytes were shown to regulate the flux of energy substrates to neurons. In pathological situations, astrocytes are activated but the consequences on brain energy metabolism are still poorly characterized. We found that local lentiviral-mediated gene transfer of ciliary neurotrophic factor (CNTF), a cytokine known to activate astrocytes, induced a stable decrease in the glycolytic flux in the rat striatum in vivo as measured by 2-[ $\left.{ }^{18} \mathrm{~F}\right]-$ 2-deoxy-D-glucose autoradiography and micro-positron emission tomography imaging. The activity of the mitochondrial complex IV enzyme cytochrome oxidase was not modified, suggesting maintenance of downstream oxidative steps of energy production. CNTF significantly increased the phosphorylation level of the intracellular energy sensor AMP-activated protein kinase (AMPK), supporting a specific reorganization of brain energy pathways. Indeed, we found that different key enzymes/transporters of fatty acids $\beta$-oxidation and ketolysis were overexpressed by CNTF-activated astrocytes within the striatum. In primary striatal neuron/astrocyte mixed cultures exposed to CNTF, the AMPK pathway was also activated, and the rate of oxidation of fatty acids and ketone bodies was significantly enhanced. This metabolic plasticity conferred partial glial and neuronal protection against prolonged palmitate exposure and glycolysis inhibition. We conclude that CNTF-activated astrocytes may have a strong protective potential to face severe metabolic insults.

Key words: astrocytes; CNTF; ketone bodies; glucose uptake; fatty acid; neuroprotection

\section{Introduction}

Neurons have high energy needs and restricted internal energy stores. They are therefore dependent on a tight regulation of energy metabolism and supply. Astrocytes have a central role in the regulation of brain energy metabolism. They send endfeet around brain capillaries (Kacem et al., 1998), which allow them to control the entry of energy substrates into brain parenchyma. Glucose is, by far, the major brain energy substrate, and astrocytes participate in matching glucose supply to neuronal energy needs (Pellerin and Magistretti, 1994; Voutsinos-Porche et al., 2003). Other energetic blood-borne substrates, such as fatty acids and ketone bodies, may also fuel brain function, depending on their availability. Astrocytes are the only cell type able to perform

Received Jan. 16, 2007; revised May 9, 2007; accepted May 14, 2007.

This work was supported by the Centre National de la Recherche Scientifique, Commissariatà l'Energie Atomique, and the "Programme Interdisciplinaire Imagerie du Petit Animal" (G.B.) and by Fonds National Suisse Grant 3100A01112119 (L.P.). We thank Dr. Gervais for providing FDG; Dr. Lebon for initial help in the MRI study; Dr. Jan for help with micro-PET; J. Chenal, A. Parent, and M. Faideau for helping with cell culture facilities; and Drs. Gavillet and Minehira-Castelli for their technical advice on experiments with ${ }^{14} \mathrm{CO}_{2}$. We acknowledge Dr. Peschanski for providing access to the biosafety level 3 laboratory. We are grateful to Dr. Prip-Buus, Dr. Murad, and Prof. Latruffe for giving us their CPT1, SCOT, and BDH antibodies, respectively.

Correspondence should be addressed to Dr. Gilles Bonvento, Service Hospitalier Frédéric Joliot, Commissariat à I'Energie Atomique, Centre National de la Recherche Scientifique, Unité de Recherche Associée 2210, 4 place du Général Leclerc, 91401 Orsay, France. E-mail: gilles.bonvento@cea.fr.

DOl:10.1523/JNEUROSCI.0174-07.2007

Copyright $\odot 2007$ Society for Neuroscience $\quad$ 0270-6474/07/277094-11\$15.00/0 $\beta$-oxidation of fatty acids in the brain (Edmond et al., 1987), a catabolic pathway that may represent up to $20 \%$ of brain oxidative energy production (Ebert et al., 2003). The rate of $\beta$-oxidation in astrocytes is under the tight control of the AMPactivated protein kinase (AMPK) (Blazquez et al., 1999), a central protein to promote a concerted regulation of many energy metabolism pathways (Hardie et al., 2006). Astrocytes also use fatty acids as precursors for the synthesis of ketone bodies that are exchanged with neurons (Auestad et al., 1991; Guzman and Blazquez, 2001). Indeed, ketone bodies also represent a major fuel for the brain in specific conditions (Sokoloff, 1973).

In response to a wide range of pathological situations, astrocytes become reactive and display a very distinct phenotype (Kalman, 2004). These phenotypic changes are likely accompanied by changes in their function that, in turn, may either be beneficial or detrimental for brain recovery; this issue remains very controversial. In addition, little is known about the metabolic consequences of astrocyte activation, particularly in vivo.

To characterize the metabolic profile of reactive astrocytes, we used our two previously described models of astrocyte activation through either lentiviral-gene transfer of the cytokine ciliary neurotrophic factor (CNTF) in the rat striatum in vivo or exposure of primary neuron astrocyte mixed cultures to recombinant CNTF (Escartin et al., 2006). CNTF induces a sustained activation of astrocytes in a large part of the striatum, whereas neurons display 
A

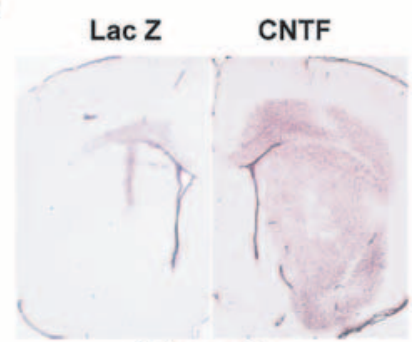

Vimentin
B

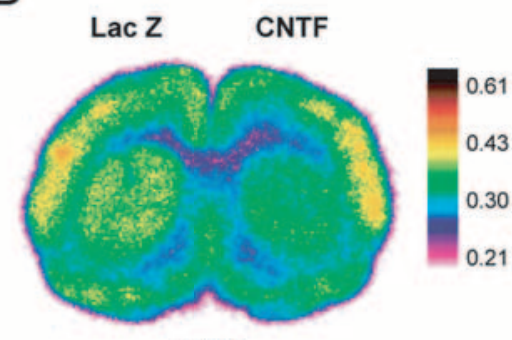

FDG
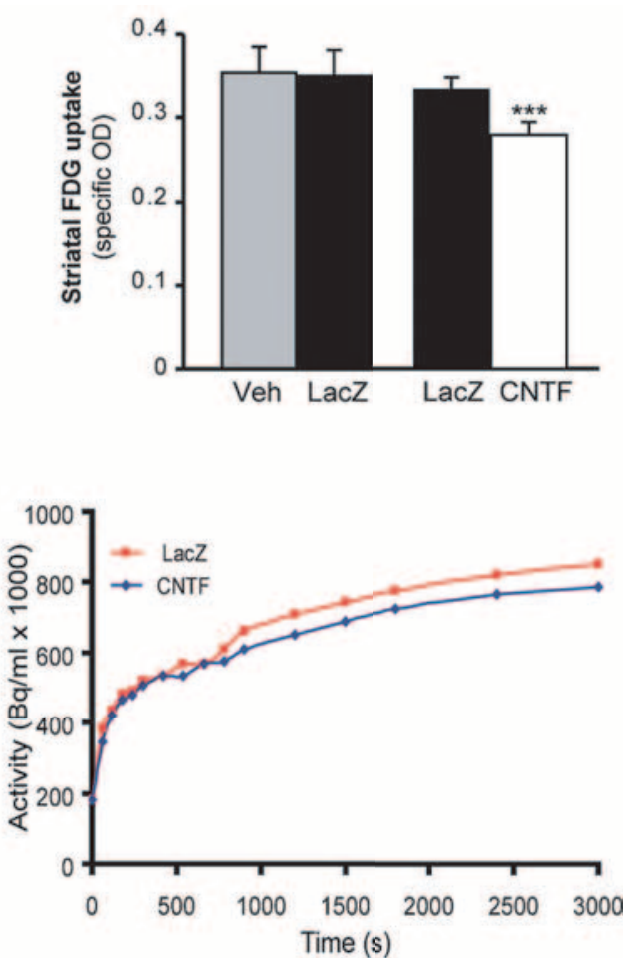

Figure 1. CNTF reduces glucose uptake in vivo. $\boldsymbol{A}$, Vimentin immunostaining of rat brain sections after intrastriatal injection of lentiviruses encoding either CNTF or LacZ. CNTF induces the expression of vimentin compared with the control striatum injected with lenti-LacZ. B, Measurement of striatal FDG uptake in vivo was performed using the autoradiographic technique. A decrease in FDG uptake (mean of -17\%) is observed in the whole lenti-CNTF-injected striatum. There is no significant difference between Veh and lenti-LacZ-injected striata for FDG uptake. C, Example of an FDG-PET scan obtained at 4 months after lenti-CNTF injection. After image reconstruction, PET scans were overlaid with anatomical magnetic resonance images for drawing regions of interest from striatum (red: LacZ; blue: CNTF). The curve represents a typical time course of FDG accumulation in both regions and shows a reduced FDG uptake in the CNTF-injected striatum. Data are expressed as mean \pm SEM; ${ }^{* * *} p<0.001 ;$ Student's paired $t$ test; $n=6$ per group.

a normal phenotype in terms of morphology, expression of neuronal proteins, and spontaneous electrophysiological activity. Many aspects of in vivo astrocyte activation are reproduced by the exposure of primary mixed cultures to CNTF in vitro (Escartin et al., 2006). Interestingly, in addition to its well known effects on brain cells, CNTF is known to modify the metabolism of peripheral organs either directly (Sleeman et al., 2003; Watt et al., 2006b) or through central effects mediated by the hypothalamus (Gloaguen et al., 1997; Xu et al., 1998; Lambert et al., 2001).

We found that CNTF-induced astrocyte activation in the striatum was associated with a marked rearrangement of metabolic features. The metabolic plasticity of CNTF-activated astrocytes significantly improved their ability to resist to metabolic insults, ultimately providing protection to neurons.

\section{Materials and Methods}

Reagents and animals. All products come from Sigma (St. Louis, MO), unless otherwise specified.

Three-month-old male Lewis rats (weight, $\sim 300$ g; IFFA Credo, L'Arbresle, France) were used for in vivo experiments. Embryos used for primary cell cultures were taken from time pregnant Sprague Dawley rats (Janvier, Le Genest, France). All surgical procedures were performed in strict accordance with the recommendations of the European Communities Council (86/609/EEC) and in conformity with the Swiss national institutional guidelines on animal experimentation.

Stereotaxic injections of lentiviruses in the rat striatum. Rats received stereotaxic injections of self-inactivated lentiviruses that encode either the human CNTF c-DNA (lenti-CNTF) with the export sequence of immunoglobulin or the $\beta$-galactosidase gene (lenti-LacZ) under the control of the mouse phosphoglycerate kinase 1 promoter, as described previously (Escartin et al., 2006). The production and characteristics of these
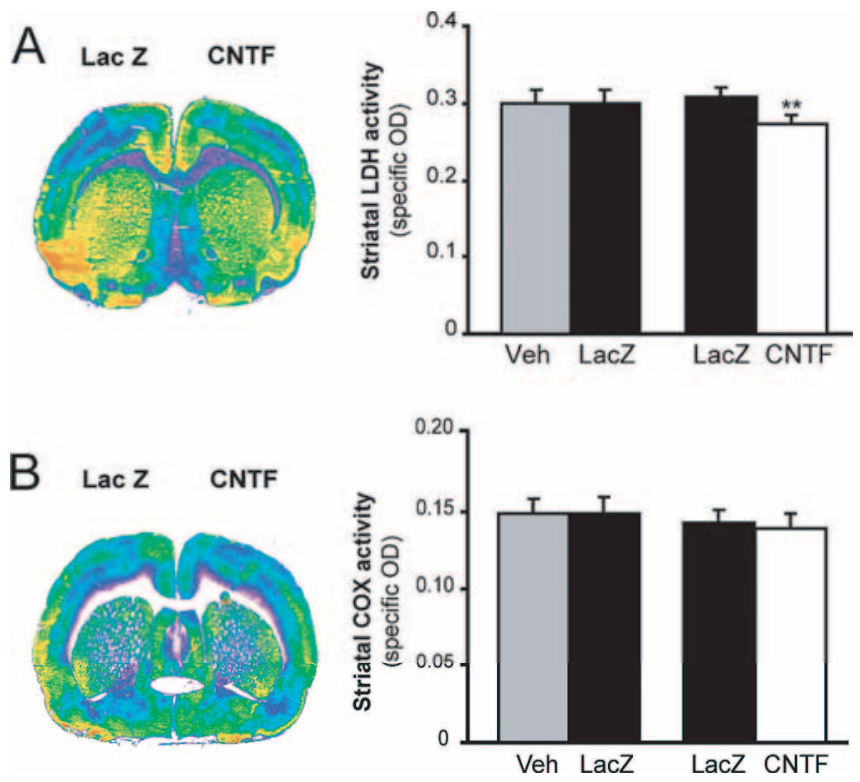

Figure 2. CNTF decreases the activity of the glycolytic enzyme LDH but does not affect oxidative phosphorylation in vivo. $A, \mathrm{LDH}$ activity is slightly decreased by $11 \%$ in the striatum injected with lenti-CNTF, as evidenced on brain sections processed for LDH histochemistry. $\boldsymbol{B}$, CNTF overexpression has no effect on striatal oxidative metabolism as shown by COX histochemistry. Data are expressed as mean $\pm S E M ; * * p<0.01$; Student's paired $t$ test; $n=6$ per group.

lentiviruses have been described previously (Escartin et al., 2006). Rats were either injected with different viruses or vehicle (Veh; PBS plus 1\% BSA) in each striatum (lenti-CNTF/lenti-LacZ and lenti-LacZ/Veh rats) or injected bilaterally with the same virus or vehicle (lenti-CTF, lenti-LacZ, and Veh 
A
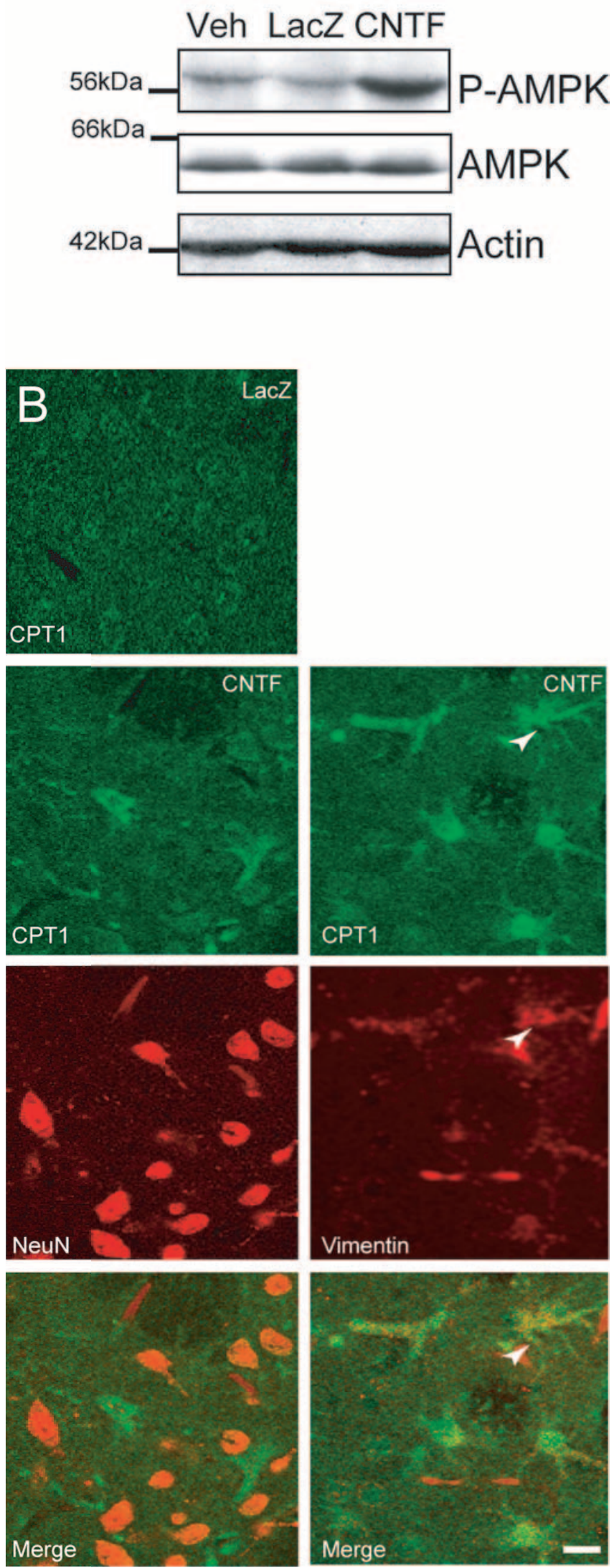

rats). Experiments were performed between $15 \mathrm{~d}$ and 6 months after infection, because the activation of astrocytes by CNTF was demonstrated to be stable over this period (Escartin et al., 2006).

Primary striatal neuron/astrocyte cultures. Primary striatal neuron/astrocyte mixed cultures were prepared from embryonic day 14 (E14) to E15 rat embryos as described previously (Escartin et al., 2006) and grown in complete Neurobasal medium [serum-free Neurobasal medium supplemented with 2\% B27 (Invitrogen, Carlsbad, CA), 1\% antibioticantifungal mixture (Invitrogen), and $0.5 \mathrm{~mm}$ L-glutamine]. Human recombinant CNTF ( $\mathrm{R} \&$ D Systems, Minneapolis, MN) was diluted in PBS and $0.1 \%$ BSA at a stock concentration of $25 \mu \mathrm{g} / \mathrm{ml}$. CNTF $(50 \mathrm{ng} / \mathrm{ml}$; dilution, 1/500) was added at $15 \mathrm{~d}$ in vitro (DIV) and at 17 DIV. Control wells were treated with vehicle (final concentration, $2.5 \mu \mathrm{g} / \mathrm{ml}$ BSA in PBS). Cell cultures were studied at 19 DIV.

Western blots. The striatum of lenti-CNTF, lenti-LacZ, or Veh rats ( $n=4-5$ per group) was rapidly dissected out on ice. Tissue samples and cell cultures were homogenized, and Western blots were made as described previously (Herard et al., 2005) using the ECL detection technique (GE Healthcare, Little Chalfont, UK). Films were scanned, and optical density was measured and normalized to actin. The antibodies used were raised against actin (1:10,000), AMPK $\alpha$ (1:500; Cell Signaling, Beverly, MA), phospho-AMPK (Thr172, 1:1000; Cell Signaling), and glucose transporter 1 (Glut-1, 1: 500; Calbiochem, La Jolla, CA).

Immunochemistry. Brains sections and cell cultures were processed for double-labeling immunochemistry as described previously (Escartin et al., 2006) and were studied with confocal microscopy using a Zeiss (Oberkochen, Germany) confocal microscope.

The antibodies used were as follows: $\beta$-hydroxybutyrate dehydrogenase (BDH, 1:250; a kind gift from Prof. Latruffe, Université de Dijon, Dijon, France), carnityl-palmityl transferase 1 (CPT1, 1:500; a kind gift from Dr. Prib-Buus, Cochin Institute, Paris, France), GFAP (1:500; Dako, Carpinteria, CA), microtubule-associated protein 2 (MAP2; 1:200), nestin (1:500), succinyl-CoA:3-oxoacid CoA-transferase (SCOT; 1:500, a kind gift from Dr. Murad, University of Texas, Austin, TX), S100 $\beta$ (1:1000), and vimentin (1:1000; Calbiochem). Vimentin and monocarboxylate transporter 1 (MCT1) immunochemistry were performed as described previously (Pierre et al., 2000; Escartin et al., 2006).

Histochemistry. Histochemistry of lactate dehydrogenase (LDH), cytochrome oxidase (COX), and $\mathrm{BDH}$ were performed according to the method of Dufour et al. (2003), Greene and Greenamyre (1995), and Bilger and Nehlig (1992), respectively. Coronal brain sections were cut from frozen brains of lenti-CNTF/lenti-LacZ and Veh/lenti-LacZ rats ( $n=7$ per group) using a cryostat and were stored immediately at $-80^{\circ} \mathrm{C}$. Six serial sections (interspace, $100 \mu \mathrm{m}$ ) from each animal were processed for histochemistry of each enzyme. Nonspecific staining was obtained by incubating successive sections (three animals per group) without substrate. Sections were digitized and striatal optical densities were measured. Specific activity was calculated as the difference between total and nonspecific staining.

Determination of glucose plasma concentrations. Lenti-CNTF, LentiLacZ, and Veh rats ( $n=4-6$ per group) were anesthetized and a catheter implanted in the femoral artery. Glucose concentration was determined on arterial blood samples using a OneTouch glucose meter (Lifescan, Milpitas, CA) after night fasting.

In vivo $2-\left[{ }^{18} \mathrm{~F}\right]$-deoxy-D-glucose uptake. Glucose uptake was assessed on lenti-CNTF/lenti-LacZ rats and Veh/lenti-LacZ ( $n=6$ per group). Rats received an intraperitoneal injection of 2-[ $\left.{ }^{18} \mathrm{~F}\right]$-2-deoxy-D-glucose (FDG; $1-1.7 \mathrm{mCi}$ in $1 \mathrm{ml}$ of normal saline) and were allowed to move freely during the experiment. Forty-five minutes after FDG injection, brains were cut into $20 \mu \mathrm{m}$ sections with a cryostat and processed for

$\leftarrow$

Figure 3. CNTF activates AMPK and increases CPT1 expression in astrocytes in vivo. A, CNTF induces a strong increase in the level of phosphorylation of AMPK without altering the total level of expression of AMPK in the rat striatum. $\boldsymbol{B}$, In the CNTF-injected striatum, cells with a strong labeling for CPT1 (green) are observed; they coexpress vimentin (red) but not the neuronal protein NeuN (red). Scale bar, $20 \mu \mathrm{m}$. Blots and images are representative of four to five animals per group. 
autoradiography. Autoradiograms were digitized and striatal optical densities were measured on 10 successive sections for each animal.

To investigate long-term metabolic effect of $\mathrm{CNTF}$, noninvasive imaging studies of glucose uptake by micro-positron emission tomography (PET; Concorde Focus200 PET scanner) were repeated at 4 weeks and 4 months after injection on lenti-CNTF/lenti-lacZ rats $(n=6)$. Rats were anesthetized with a mixture of ketamine $(15 \mathrm{mg} / \mathrm{kg})$ and xylazine $(1.5 \mathrm{mg} / \mathrm{kg})$, and a catheter was introduced into the tail vein for administration of FDG (2-3.5 mCi). Animals were mounted onto a home-made stereotaxic frame compatible with PET acquisition to allow accurate repositioning of the animal in longitudinal studies. Dynamic accumulation of radioactivity into the brain was acquired for 60 min. After image reconstruction, PET images were overlaid with anatomical magnetic resonance images for drawing three-dimensional (3D) regions of interest from the striatum. Magnetic resonance imaging studies were conducted in a whole-body $3 \mathrm{~T}$ system with a homebuilt, transmit/receive bird-cage coil. Inversion recovery fast spin echo $\mathrm{T} 2$-weighted $3 \mathrm{D}$ images were recorded using the following parameters: repetition time, $2000 \mathrm{~ms}$; echo time (TE), 10.1 $\mathrm{ms}$; weighted TE, $79.4 \mathrm{~ms}$; rare factor, 16; field of view, $3.84 \times 3.84 \times 3.84 \mathrm{~cm}^{3}$; matrix, $128 \times$ $128 \times 128$; resolution, $300 \times 300 \times 300 \mu \mathrm{m}^{3}$.

In vitro oxidation of $\left[{ }^{14} \mathrm{C}\right]$-3-hydroxybutyrate to ${ }^{14} \mathrm{CO}_{2}$. Mixed cultures grown on plastic petri dishes (diameter, $35 \mathrm{~mm}$ ) were switched to DMEM supplemented with $1 \mathrm{~mm}$ D-glucose, $5 \mathrm{~mm}$ HEPES, $7.5 \mathrm{~mm}$ $\mathrm{NaHCO}_{3}$, and antibiotic-antifungal mixture $\left(\mathrm{DMEM}_{1}\right)$ for $1 \mathrm{~h}$. Cells were then incubated in $\mathrm{DMEM}_{1}$ with $2 \mathrm{~mm}$ 3-hydroxybutyrate (BHB) plus $0.4 \mu \mathrm{Ci} / \mathrm{ml} \quad\left[{ }^{14} \mathrm{C}\right]-\mathrm{BHB} \quad$ (specific activity, $35 \mathrm{mCi} / \mathrm{mmol}$; PerkinElmer, Boston, MA) in hermetic vials. After $50 \mathrm{~min}, \mathrm{CO}_{2}$ was released from the medium through acidification by $0.5 \mathrm{ml}$ of $0.2 \mathrm{M} \mathrm{HCl}$. $\mathrm{CO}_{2}$ was trapped by $1 \mathrm{ml}$ Carbosorb (PerkinElmer), added in the chamber outside the dish. After $1 \mathrm{~h}$ of equilibration, $450 \mu \mathrm{l}$ of Carbosorb was taken in duplicate for scintillation counting. Background level was evaluated on cells lysed by $1 \%$ Triton X-100 before incubation in the same medium. The inhibitor of MCTs $\alpha$-cyano-4-hydroxycinnamate (4-CIN) was added $20 \mathrm{~min}$ before incubation with radioactive $\mathrm{BHB}$ at a concentration of $1.5 \mathrm{~mm}$ and maintained during the $50 \mathrm{~min}$ incubation at a concentration of $3 \mathrm{~mm}$. The production of ${ }^{14} \mathrm{CO}_{2}$ was normalized to the protein content of the dish and the background was subtracted. Experiments were made on three to four dishes per treatment and repeated on four separate cultures.

In vitro oxidation of $\left[{ }^{14} \mathrm{C}\right]$-palmitate to ${ }^{14} \mathrm{C}$-intermediate metabolites and ${ }^{14} \mathrm{CO}_{2}$. The same experiment was performed using $\left[{ }^{14} \mathrm{C}(\mathrm{U})\right]-$ palmitate as a substrate $(0.4 \mu \mathrm{Ci} / \mathrm{ml}$; specific activity, $850 \mathrm{mCi} / \mathrm{mmol}$; PerkinElmer). Labeled palmitate was first mixed with albumin-bound cold palmitate (BSA to palmitate molar ratio, $\sim 5: 1$ ) and then added to $\mathrm{DMEM}_{1}$ and $0.5 \mathrm{~mm}$ L-carnitin (final concentration, $0.2 \mathrm{~mm}$ total palmitate). At the end of a $2 \mathrm{~h}$ incubation period, the medium was acidified with perchloric acid to precipitate proteins and albumin-bound palmitate and to isolate the acid-soluble metabolites of palmitate (i.e., mainly ketone bodies in addition to Krebs cycle intermediates and short-chain fatty acids) (Blazquez et al., 1998). The acidified medium was collected, centrifuged, and the radioactivity found in the supernatant was counted. Sodium etomoxir, the inhibitor of CPT1, and 4-CIN were used at a concentration of $40 \mu \mathrm{M}$ and $3 \mathrm{~mm}$, respectively. They were added $20 \mathrm{~min}$ before incubation with ${ }^{14} \mathrm{C}$-palmitate and maintained throughout the incubation period. Experiments were made on two to five wells per treatment and repeated on two to four separate cultures.

Exposure to palmitate. After a $72 \mathrm{~h}$ treatment with Veh or CNTF, cells were exposed to $0.2 \mathrm{~mm}$ BSA-bound palmitate plus $0.5 \mathrm{~mm} \mathrm{~L}$-carnitine for
$24 \mathrm{~h}$ in complete Neurobasal medium. Some cells were exposed to palmitate in the presence of $2 \mathrm{~mm} 4$-pentenoate to inhibit $\beta$-oxidation. Astrocytes and neurons were labeled with GFAP and MAP2, respectively. 4-pentenoate and BSA had no effect by themselves on the survival of astrocytes and neurons (data not shown). Images were obtained from two to three wells and repeated on three independent cultures.

Exposure to iodoacetate. After a $72 \mathrm{~h}$ treatment with Veh or CNTF, the culture medium was collected, stored at $37^{\circ} \mathrm{C}$, and replaced with $0.4 \mathrm{~mm}$ iodoacetate (IAA) in complete Neurobasal medium, in the absence of CNTF or Veh. After $10 \mathrm{~min}$, cultures were returned to their previous medium for $24 \mathrm{~h}$. Control cultures were also subjected to this change of medium. 4-CIN ( $3 \mathrm{~mm}$ final concentration) was added during the incubation with IAA and the $24 \mathrm{~h}$ recovery period. 4 -CIN alone did not have any detectable effect on the survival of astrocytes and neurons (data not shown). Total cell survival was quantified by counting DAPI-positive nuclei on 13-21 randomly chosen fields taken at the $20 \times$ objective using a motorized Zeiss Axioplan2 imaging microscope (Carl Zeiss, Vesinet, France). Images were obtained from two to three wells and repeated on three to six independent cultures for each treatment.

Statistical analysis. Results are expressed as mean values \pm SEM. Statistical analysis included unpaired Student's $t$ test and one-way ANOVA followed by a post hoc $F$ Scheffé test (when comparing two or three groups, respectively). Student's paired $t$ test was performed for left-right comparisons in vivo. The significance level was set at $p<0.05$.

\section{Results}

\section{CNTF decreases striatal glycolysis in vivo}

We measured brain glucose uptake in the striatum using autoradiography after injection of FDG. FDG uptake was not modified by the injection of the lentivirus itself (no difference between lenti-LacZ and Veh) (Fig. $1 B$ ). In the striatum injected with a lentivirus encoding CNTF (lenti-CNTF), we found a consistent decrease in FDG uptake compared with the contralateral striatum injected with lenti-LacZ, 2 months after injection $(-17 \%$; $p<0.001$; Student's paired $t$ test) (Fig. $1 B$ ). The spatial extent of decreased FDG uptake matched well with the location of activated astrocytes as evidenced by vimentin expression (Fig. $1 A$ ). 

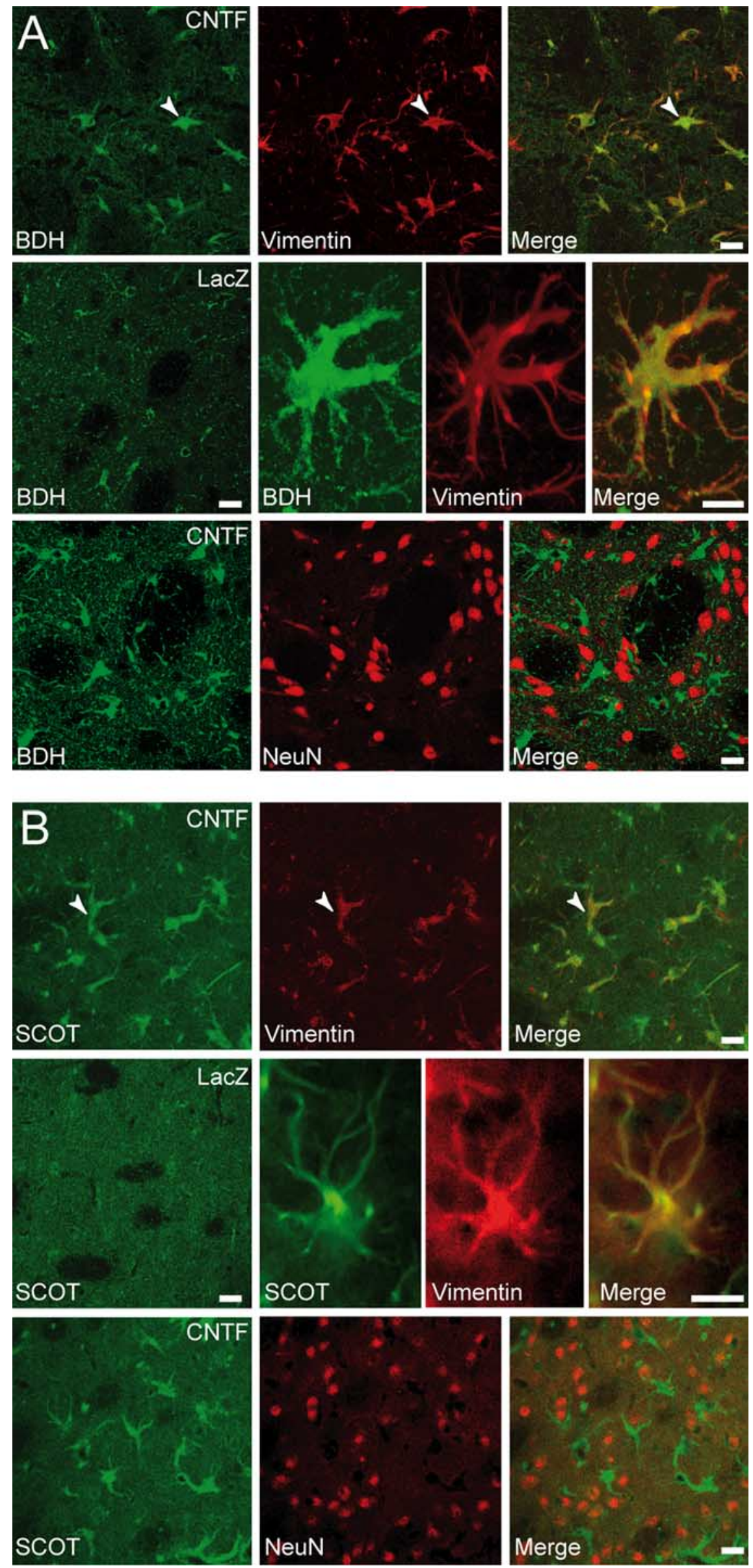

We also used micro-PET imaging to perform a repeated analysis of FDG uptake in the living animal. We observed a significant decrease in FDG uptake in the striatum injected with lenti-CNTF (Fig. 1C). This decrease was consistently present over a period of 4 months, demonstrating the stability of the metabolic change. The striatal level of expression of the endothelial and astroglial Glut-1 measured by Western blot, was not different between groups ( $p=0.92$; ANOVA; data not shown), indicating a preserved capacity of glucose transport across the blood-brain barrier. The activity of LDH (the enzyme that catalyzes the reversible conversion of lactate to pyruvate) was studied by histochemistry on brain sections from the same rats used for FDG uptake. LDH activity (pyruvate-to-lactate reaction) was reduced by $11 \%$ in the lentiCNTF injected striatum $(p<0.01$; Student's paired $t$ test) (Fig. $2 A$ ), which is consistent with a reduction in the glycolytic flux with CNTF. Importantly, CNTF overexpression had no detectable effects on peripheral metabolism: rats injected bilaterally with lenti-CNTF in the striatum had a normal weight gain during 2 months compared with control groups and had normal blood glucose levels (arterial glycemia after night fasting in the following groups: lenti-CNTF, $1.71 \pm 0.04 \mathrm{~g} / \mathrm{L}$; lentiLacZ, $1.68 \pm 0.08$; Veh, $1.69 \pm 0.07$; $p=0.95$; ANOVA).

To assess whether CNTF induced a general decrease in striatal oxidative metabolism, we measured the activity of the mitochondrial complex IV enzyme COX, using histochemistry on brain sections. Quantification of specific COX activity in the striatum showed no difference between groups ( $p=0.44$ between lentiCNTF and lenti-LacZ; $p=0.92$ between Veh and lenti-LacZ; Student's paired $t$ test) (Fig. 2B).

CNTF activates AMPK and increases CPT1 expression in astrocytes in vivo As AMPK plays a pivotal role in the coordinate regulation of several energy metabolism

\section{$\leftarrow$}

Figure 5. CNTF-activated astrocytes overexpress $\mathrm{BDH}$ and SCOT.A, Stellate cells with an intense BDH staining (green) are only visible in the lenti-CNTF striatum (arrowhead). BDH colocalizes with vimentin (red) but not with the neuronal protein NeuN (red). B, SCOT (green), the second enzyme on the ketone bodies pathway, is also overexpressed by CNTF-activated astrocytes that coexpress vimentin (red, arrowhead) but not by NeuNpositive neurons (red). Images are representative of four to five animals per group. Scale bars: $20 \mu \mathrm{m}$; enlarged pictures, $10 \mu \mathrm{m}$. 

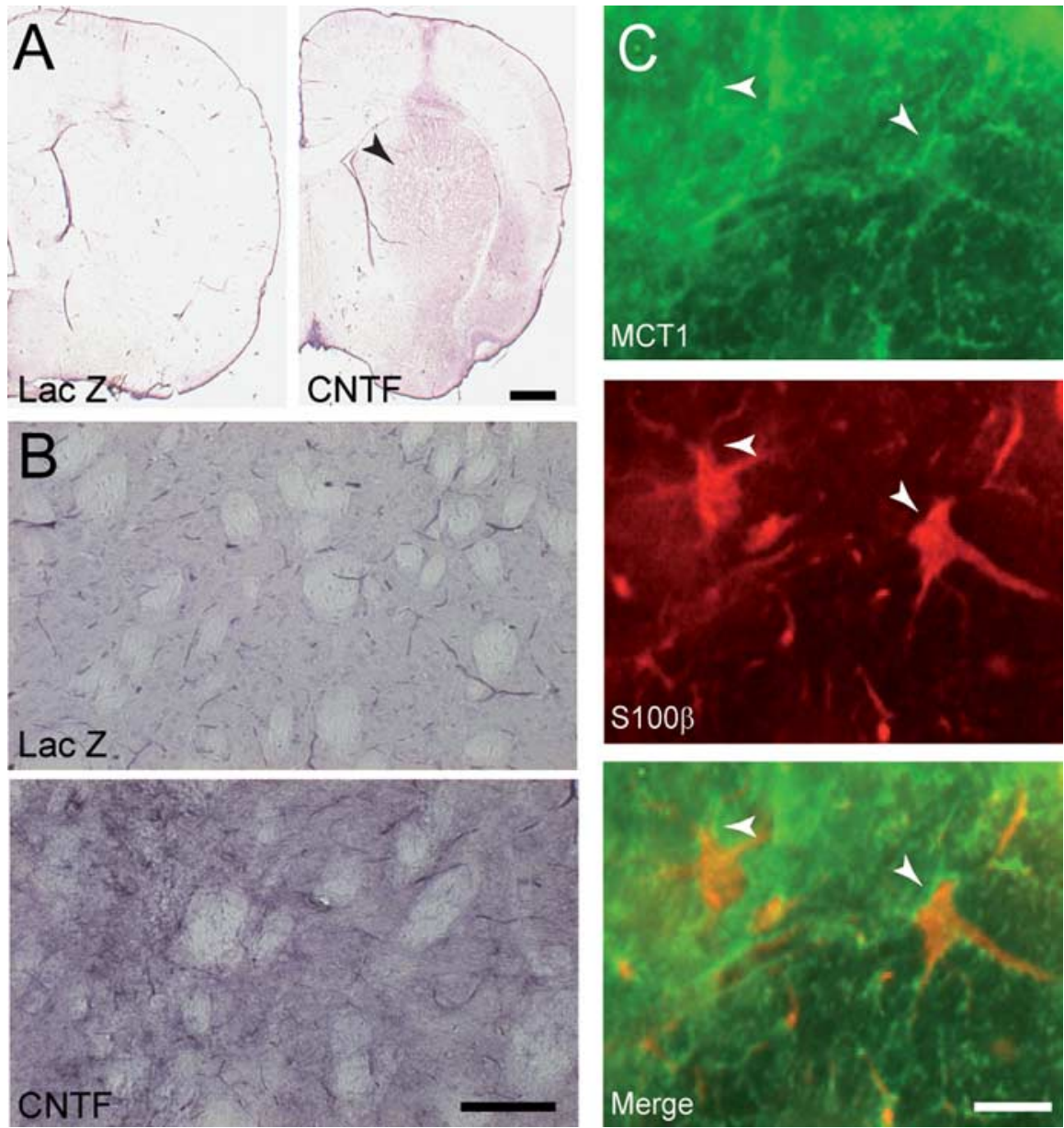

Figure 6. CNTF-activated astrocytes overexpress MCT1. A, B, MCT1 is overexpressed in the striatum of rats of the lenti-CNTF group in the region where astrocytes are activated (arrowhead). C, MCT1 immunoreactivity is found around S100 $\beta$-positive astrocytes (arrowhead), as expected for a plasma membrane transporter. Images are representative of four to five animals per group. Scale bars: $A, 1 \mathrm{~mm} ; \boldsymbol{B}, 100 \mu \mathrm{m} ; C, 20 \mu \mathrm{m}$.

pathways within the cell (Hardie et al., 2006), we determined the level of phosphorylated AMPK (active form) in the striatum of lentiCNTF-injected rats. We found that CNTF significantly increased the expression of phosphorylated AMPK $(+280 \% ; p<0.001$ vs lentiLacZ and Veh groups; Scheffe's test) (Fig. 3A), demonstrating a robust activation of the AMPK pathway.

In astrocytes, AMPK regulates the activity of $\mathrm{CPT}$, a ratelimiting enzyme for $\beta$-oxidation of fatty acids (Blazquez et al., 1999). In rats injected with lenti-LacZ, the expression of CPT1 (the predominant brain isoform) was low and restricted to the neuropil. In the lenti-CNTF striatum, many stellate cells displaying an intense staining became apparent (Fig. 3B). These cells coexpressed vimentin, a marker of activated astrocytes, but not the neuronal protein $\mathrm{NeuN}$ (Fig. $3 B$ ), demonstrating that $\mathrm{CPT} 1$ is specifically overexpressed in CNTF-activated astrocytes.

\section{CNTF-activated astrocytes overexpress key proteins of the ketone bodies metabolic pathway in vivo}

Using histochemistry, we next characterized the activity of $\mathrm{BDH}$, a key enzyme of the ketone bodies metabolic pathway, another alternative energy-producing pathway. Whereas no labeling was observed in the lenti-LacZ injected striatum, numerous cells displaying an intense staining were observed in the contralateral lenti-CNTF injected striatum. These cells were sometimes in close contact with brain capillaries (Fig. 4). Dual-labeling exper- iments were performed to unambiguously identify these cells. We found a specific colocalization of $\mathrm{BDH}$ with vimentin, but not with NeuN (Fig. 5A). Similarly, SCOT, another enzyme of the pathway responsible for the oxidation of ketone bodies, was also specifically upregulated by CNTFactivated astrocytes in vivo (Fig. $5 B$ ). Because ketone bodies uptake into cells is dependent on MCTs, we studied the expression of MCT1, one of the main astrocytic MCTs (Pierre et al., 2000). MCT1 was overexpressed in a large part of the striatum injected with lenti-CNTF (Fig. $6 A$ ). The intensity of the labeling was uniformly increased in the neuropil (Fig. 6B). Using double labeling, we found that MCT1 immunoreactivity was present at the surface of S100 $\beta$-positive cells (Fig. $6 C)$, confirming that CNTF exposure induces MCT1 overexpression at the astrocyte plasma membrane. The pattern of expression of the neuronal transporter MCT2 was not significantly modified by CNTF (data not shown).

\section{CNTF increases the rate of oxidation of} fatty acids and ketone bodies in vitro Measurement of oxidative rate on intact cells is classically performed in vitro by quantification of released ${ }^{14} \mathrm{CO}_{2}$ produced from ${ }^{14} \mathrm{C}$-labeled substrates through the TCA cycle. We took advantage of our previously described in vitro model of CNTFmediated activation of astrocytes (Escartin et al., 2006) to assess whether fatty acids and ketone bodies were indeed used as alternative energetic sources by CNTFactivated astrocytes. First of all, we found that CNTF induced a significant increase in the level of phosphorylated AMPK compared with Veh-treated primary striatal cultures $(+360 \%$; $p<$ 0.001; unpaired $t$ test) (Fig. 7A), an observation consistent with the effect of CNTF obtained in vivo. To test the hypothesis that oxidation of fatty acids was enhanced by CNTF, we quantified ${ }^{14} \mathrm{C}$-palmitate oxidation to ${ }^{14} \mathrm{CO}_{2}$ and the formation of ${ }^{14} \mathrm{C}$ intermediate metabolites (such as TCA cycle intermediates, ketone bodies, etc.) in cultured cells. CNTF treatment significantly increased ${ }^{14} \mathrm{CO}_{2}$ production from $0.2 \mathrm{~mm}$ palmitate by $\sim 100 \%$ $(p<0.001$; Scheffé's test) (Fig. 7B). The level of labeled acidsoluble metabolites was not modified by CNTF, suggesting that CNTF enhanced the flux of fatty acids through $\beta$-oxidation and the TCA cycle without increasing the storage of intermediate metabolites. Inhibition of CPT1 by $40 \mu \mathrm{M}$ etomoxir reduced the production of ${ }^{14} \mathrm{CO}_{2}$ by $77 \%$ and the accumulation of ${ }^{14} \mathrm{C}$-acid soluble metabolites by $43 \%$ relative to controls (data not shown). This result confirms that palmitate oxidation is dependent on its uptake into mitochondria by CPT1, which is expressed exclusively by astrocytes, at a high level in vitro (data not shown).

Astrocytes can metabolize fatty acids to produce ketone bodies and export them to neurons (Guzman and Blazquez, 2001). To test the hypothesis of a cellular compartmentalization between astrocytes and neurons to achieve complete oxidation of fatty acids (i.e., $\beta$-oxidation and ketogenesis in astrocytes, ketol- 
ysis in neurons), we studied the consequences of ketone bodies uptake blockade by $3 \mathrm{~mm}$ 4-CIN (an inhibitor of MCTs) on the oxidation of ${ }^{14} \mathrm{C}$-palmitate. The inhibition of ketone bodies uptake did not reduce ${ }^{14} \mathrm{CO}_{2}$ production $(p=0.99$ between Veh and Veh plus 4 -CIN and $p=0.91$ between CNTF and CNTF plus 4-CIN; Scheffé's test) and did not increase the level of labeled intermediate metabolites in any group $(p=$ 0.32; ANOVA) (Fig. $7 B$ ), suggesting that full fatty acids oxidation occurs within the same cell (most likely CNTF-activated astrocytes that express CPT1).

The rate of ketone bodies oxidation was evaluated by quantifying ${ }^{14} \mathrm{CO}_{2}$ production from ${ }^{14} \mathrm{C}$-BHB in cell cultures. CNTF treatment significantly increased ${ }^{14} \mathrm{CO}_{2}$ production from $2 \mathrm{~mm}$ BHB by $30 \%(p<$ 0.05; unpaired $t$ test) (Fig. $7 C$ ). Inhibition of MCT transporters by $3 \mathrm{~mm} 4$-CIN decreased ${ }^{14} \mathrm{CO}_{2}$ production by $76 \%$ (data not shown), demonstrating that $\mathrm{BHB}$ oxidation was mainly dependent on its uptake into cells and mitochondria by MCTs. MCT1 was expressed at a relatively low level in culture and CNTF significantly increased the expression of MCT1 in astrocytes (Fig. 7D), consistent with the previously observed effect in vivo.

CNTF-activated astrocytes can handle toxic levels of extracellular palmitate and are more resistant to

glycolytic inhibition

Fatty acid accumulation occurs in several pathological conditions and is toxic to brain cells (Bazan, 1970, 1971). In particular, prolonged exposure to palmitate induces the death of astrocytes, via the intracellular production of the pro apoptotic agent ceramide (Blazquez et al., 2000). We found that addition of $0.2 \mathrm{~mm}$ palmitate for $24 \mathrm{~h}$ induced marked morphological changes in Vehtreated astrocytes (disappearance of thin processes and formation of globular endings) and astrocyte degeneration but had no detectable effect on neurons (Fig. 8). In contrast to control astrocytes, CNTF-treated astrocytes were unaffected by palmitate (Fig. 8 ). However, palmitate was toxic to CNTFactivated astrocytes when $\beta$-oxidation was blocked by $2 \mathrm{~mm} 4$-pentenoate.

The ability of CNTF-activated astrocytes to use alternative energetic substrates besides glucose may also increase their resistance to a defect in glucose use. Treatment with the glycolysis inhibitor IAA $(0.4 \mathrm{~mm})$ for $10 \mathrm{~min}$ triggered a massive cellular degeneration in vitro after $24 \mathrm{~h}$ (83\% reduction; $p<0.001$ between Veh and Veh plus IAA; Scheffé's test) (Figs. 9, 10). Neurons and astrocytes were affected and remaining astrocytes displayed condensed, patchy processes with intense GFAP staining (Fig. 9). CNTF-activated astrocytes were markedly more resistant to glyco-
A

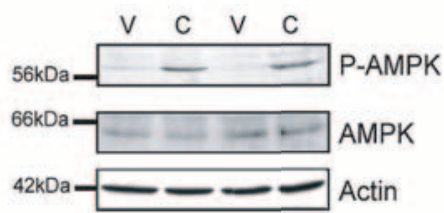

B
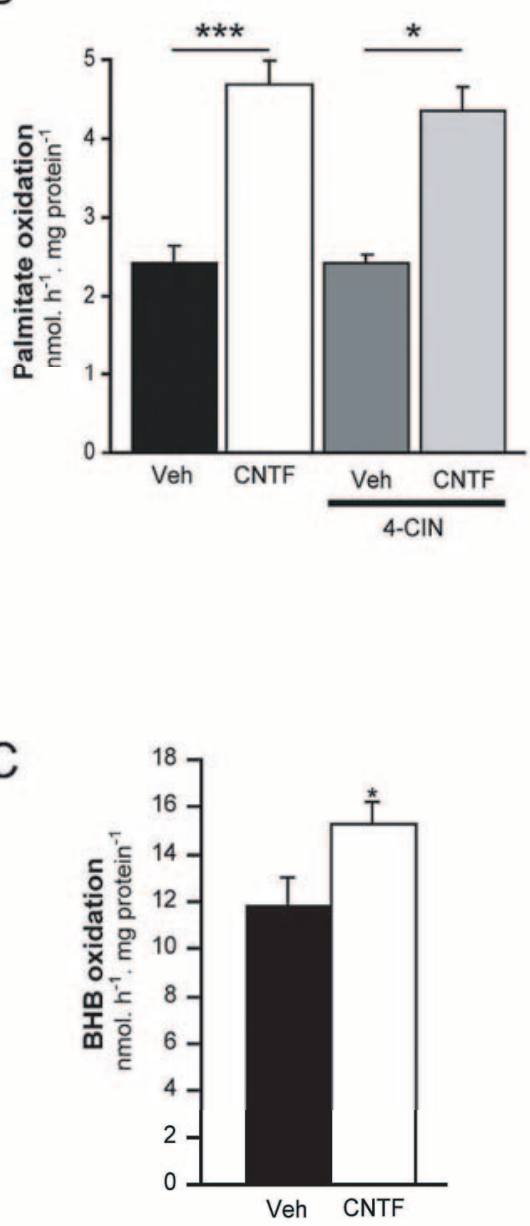
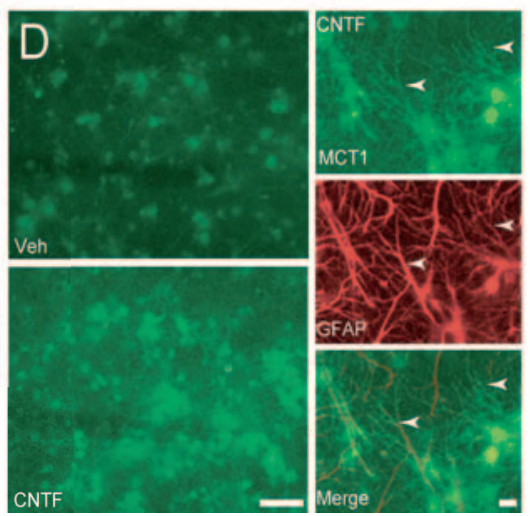

Figure 7. CNTF activates AMPK and increases the rate of fatty acids and ketone bodies oxidation in mixed cultures. $\boldsymbol{A}$, The level of phosphorylation of AMPK is significantly increased in CNTF-treated cultures (C) compared with Veh (V). Blots are representative of two to three wells per treatment from three independent cultures. $\boldsymbol{B}$, Fatty acids oxidation was evaluated by measuring the production of ${ }^{14} \mathrm{CO}_{2}$ and acid-soluble metabolites from ${ }^{14} \mathrm{C}$-palmitate. CNTF increases ${ }^{14} \mathrm{C}$-palmitate oxidation to $\mathrm{CO}_{2}\left({ }^{* * *} p<\right.$ $0.001 ;{ }^{*} p<0.05$; Scheffé's test). On the contrary, the rate of palmitate oxidation to intermediate metabolites is not altered by CNTF. Blockade of ketone bodies uptake by $3 \mathrm{~mm} 4$-CIN did not interfere with CNTF effects, suggesting that complete oxidation of fatty acids occurs within the same cell (i.e., astrocytes). Results are means \pm SEM of two to five dishes per group in two to four separate cultures. C, CNTF significantly increases ${ }^{14} \mathrm{C}-\mathrm{BHB}$ oxidation to ${ }^{14} \mathrm{CO}_{2}\left({ }^{*} p<0.05\right.$ vs Veh; Student's $t$ test). Results are means \pm SEM of three to four dishes per group in four separate cultures. D, MCT1 (green) is overexpressed in CNTF-treated cultures. MCT1 is expressed on the processes of GFAP-positive astrocytes (arrowhead). Images are representative of three wells per treatment on three independent cultures. Scale bars (in D): left, $50 \mu \mathrm{m}$; right, $20 \mu \mathrm{m}$.

lytic inhibition and had a normal morphology (Fig. 9). This improved survival of astrocytes was accompanied by a rescue of MAP2positive neurons present in the dish $24 \mathrm{~h}$ after IAA exposure (Fig. 9) so that the total number of living cells was similar to control (Fig. 10). To evaluate whether CNTF protective effects involved the uptake of alternative substrates that bypass the glycolysis block (such as ketone bodies), we used 4-CIN to inhibit MCTs and the uptake of monocarboxylates. The survival of CNTF- 
Control
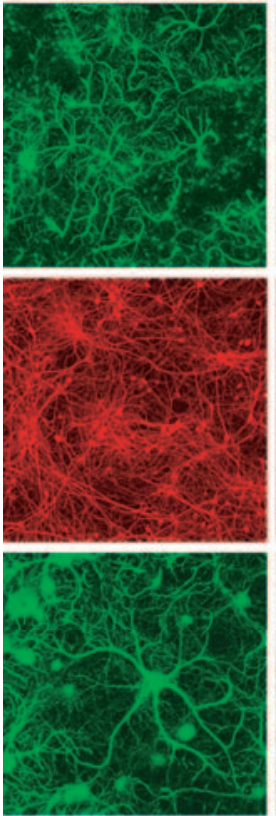

Veh
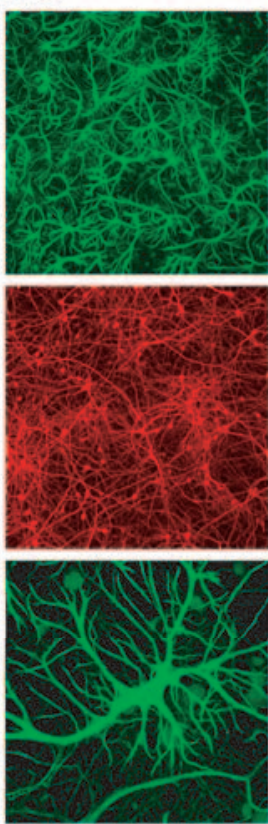

CNTF
Palmitate
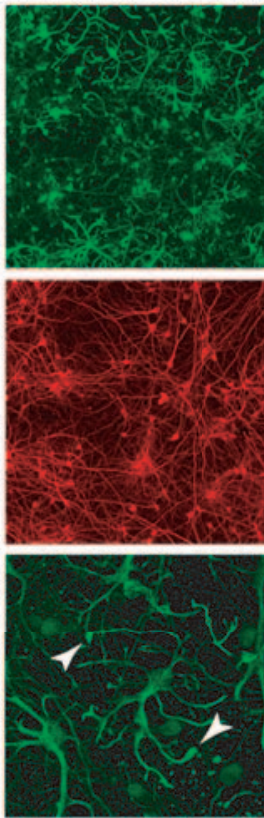

Veh

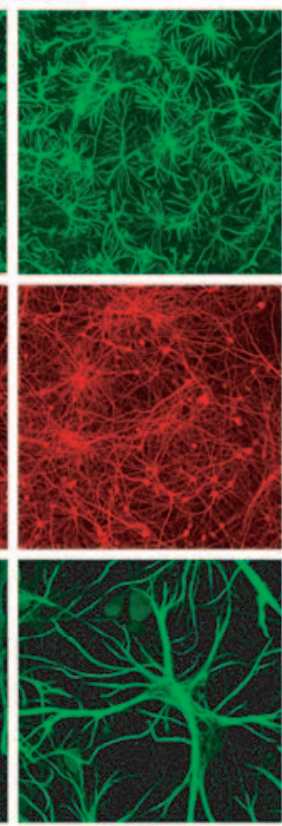

CNTF

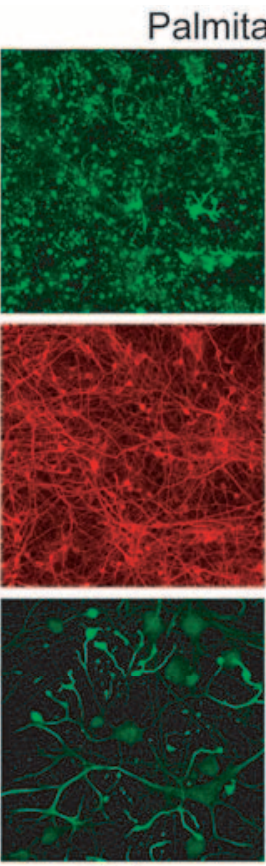

Veh
GFAP
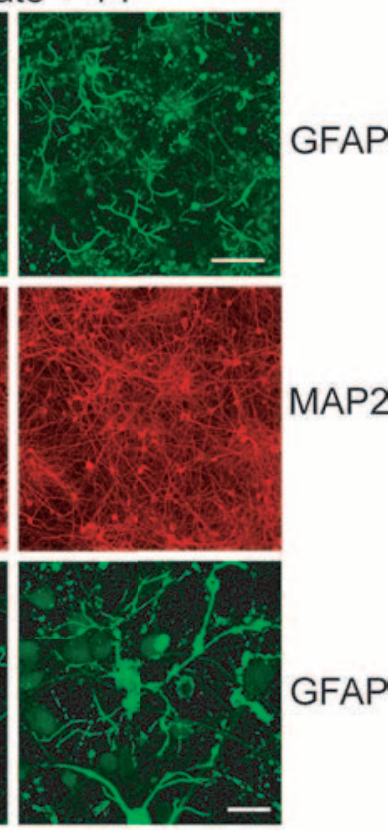

CNTF

Figure 8. CNTF-activated astrocytes handle toxic extracellular palmitate. Exposure to $0.2 \mathrm{~mm}$ BSA-bound palmitate is toxic to Veh-treated astrocytes (labeled for GFAP, green), but has no detectable effect on MAP2-positive neurons (red). Veh-treated astrocytes exposed to palmitate have a lower density than controls (CTR) and display morphological abnormalities, visible at higher magnification as fewer thin processes and globular endings (arrowhead). In CNTF-treated cultures, palmitate has no detectable effect on GFAP-positive astrocytes. When $\beta$-oxidation is inhibited by $2 \mathrm{~mm}$ 4-pentenoate (4-P), CNTF-activated astrocytes are no longer resistant to palmitate exposure. Images are representative of results obtained in two to three wells from three independent cultures. Scale bars: top, $50 \mu \mathrm{m}$; bottom, $10 \mu \mathrm{m}$

\section{Control}
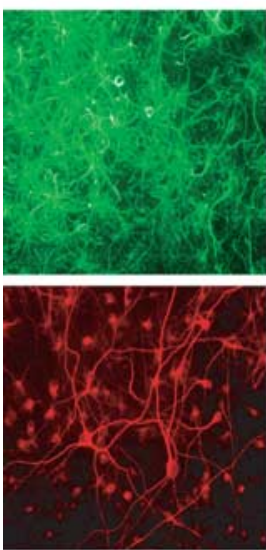

Veh
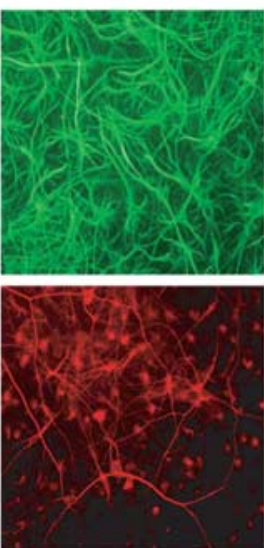

CNTF
IAA
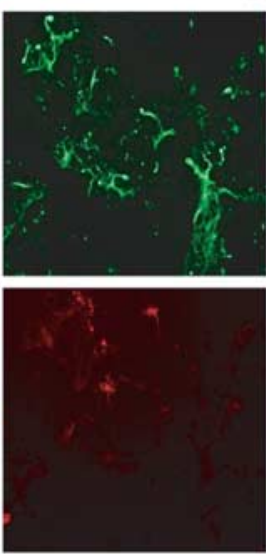

Veh
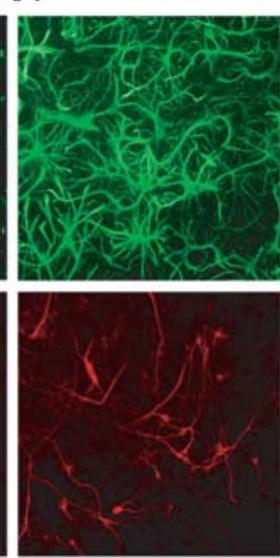

CNTF

$I A A+4-C I N$

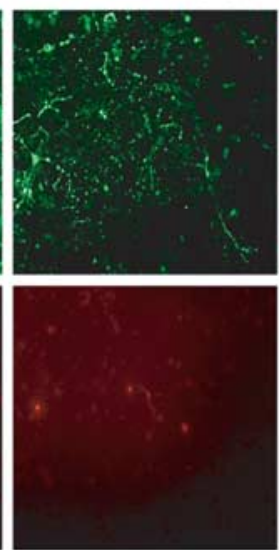

Veh

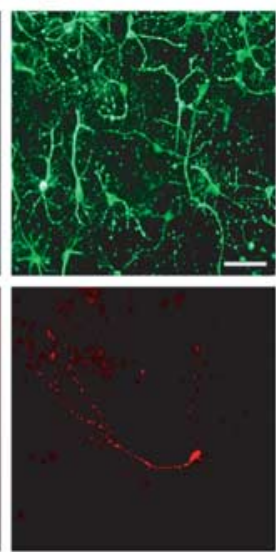

CNTF

GFAP

MAP2

Figure 9. CNTF-activated astrocytes are more resistant to glycolysis inhibition. In Veh-treated cultures, exposure to $0.4 \mathrm{~mm}$ IAA for 10 min triggers a massive degeneration of GFAP-positive astrocytes (green) and MAP2-positive neurons (red) at $24 \mathrm{~h}$ compared with control conditions. In CNTF-treated cultures, the rate of survival is markedly improved for astrocytes, and neuronal dendrites are less dystrophic. In the presence of $3 \mathrm{~mm} 4$-CIN (IAA plus 4-CIN), CNTF looses its protective effects, and both neurons and astrocytes display a pathological morphology and a low survival rate. Images are representative of results obtained in two to three wells from three to six independent cultures. Scale bar, $50 \mu \mathrm{m}$.

treated astrocytes and neurons was significantly reduced in the presence of 4 -CIN ( $p<0.001$ between CNTF plus IAA and CNTF plus IAA plus 4-CIN; Scheffé's test) (Figs. 9, 10).

Importantly, before exposure to IAA or palmitate, CNTFtreatment did not show any protective or proliferative effects on cultured cells that could explain this improved survival rate. CNTF had no significant effect on total cell densities ( $p=0.97$ between CNTF- and Veh-treated wells in control conditions; Scheffe's test) (Fig. 10), neuronal and astrocytic densities (Figs. 8, 9, controls), and total content in proteins (data not shown). In addition, CNTFactivated astrocytes were more resistant to IAA and palmitate even when CNTF was removed from the medium during the $24 \mathrm{~h}$ exposure to palmitate and during the $24 \mathrm{~h}$ period recovery after IAA exposure (data not shown).

\section{Discussion}

CNTF-mediated activation of astrocytes involves marked changes in their metabolic profile

We found that activation of astrocytes by CNTF induced stable changes in the regulation of striatal energy metabolism in vivo, including a reduction of the glycolytic flux with maintenance of downstream oxidative steps of energy production. This result 


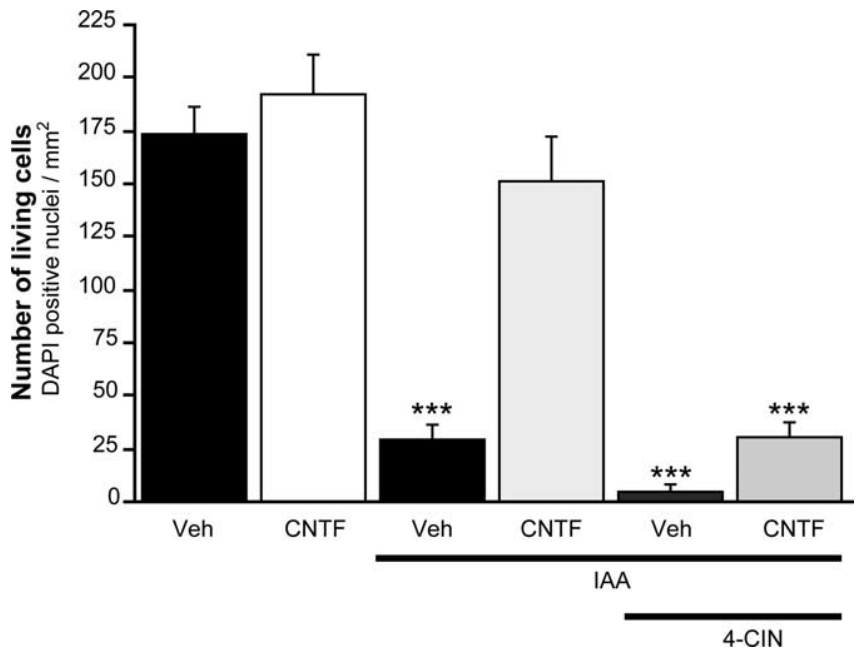

Figure 10. Quantification of CNTF protective effects against IAA. Cell counts confirm that CNTF treatment confers strong protection against IAA, an effect that is mainly dependent on MCT transporters. Data are expressed as mean \pm SEM; ${ }^{* * *} p<0.001$ versus control Veh; CNTF and CNTF plus IAA; Scheffé's test. Data are from three wells per condition in two separate cultures.

suggested that alternative pathways may have been activated to fuel the mitochondrial electron transfer chain. Our observation that CNTF activated AMPK, a central regulator of energy metabolism, both in vivo and in vitro, reinforced this idea. In the brain, fatty acids and ketone bodies can replace glucose as the major source of energy in specific conditions. Astrocytes are the only brain cell type that display a high rate of fatty acid utilization and ketogenesis (Auestad et al., 1991; Guzman and Blazquez, 2001) under the control of AMPK (Blazquez et al., 1999). We found that CNTF activated AMPK and increased the expression of CPT1 (the rate-limiting enzyme for $\beta$-oxidation of fatty acids) in astrocytes. We cannot rule out that AMPK was also activated in other cell types, although changes in metabolic enzymes or transporters were restricted to astrocytes. Interestingly, it has been recently shown that the antiobesogenic effects of CNTF resulted from the stimulation of fat oxidation directly in skeletal muscle via an activation of AMPK and an increased expression of CPT1 (Watt et al., 2006b). Our observation that CNTF increased the astrocytic expression of MCT1, SCOT, and BDH, three key enzymes/ transporters of the ketone bodies metabolic pathway, strongly supported the hypothesis that ketolysis in astrocytes was also chronically enhanced by CNTF. We next provided functional evidence that both the activity of $\mathrm{BDH}$ in vivo and the rate of oxidation of fatty acids and ketone bodies in vitro were markedly enhanced by CNTF, indicating that CNTF-activated astrocytes do use ketone bodies and fatty acids as alternative energetic substrates. We have tested the hypothesis of a cellular compartmentalization between astrocytes and neurons for a complete oxidation of fatty acids and shown that it did not involve ketone bodies exchange between astrocytes and neurons.

Altogether our results suggest that activation of astrocytes by CNTF involved a specific reorganization of their metabolic profile (Fig. 11) rather than a general enhancement of their oxidative capacity, as it was proposed previously for reactive astrocytes surrounding brain lesions (Smith, 1963; Al-Ali and Robinson, 1982). Interestingly, we have previously shown that CNTFactivated astrocytes have enlarged endfeet around striatal capillaries (Escartin et al., 2006). This morphological alteration may have an impact on blood flow and energetic supply and could represent an additional mechanism for CNTF-activated astrocytes to modulate brain metabolism.

The metabolic profile of CNTF-activated astrocytes is highly reminiscent of the immature brain that metabolizes ketone bodies and fatty acids at a high rate before weaning (Edmond, 1992). However, in the adult, these substrates are not as abundant as during the lactating period, and they are much less abundant than glucose. Nevertheless, given the basal concentration of free palmitate in the adult brain parenchyma $(\sim 0.06 \mathrm{~mm})$ (Lipton, 1999 ), its oxidative rate in vitro (comparable with glucose oxidative rate) (Edmond, 1992) and the ATP yield of $\beta$-oxidation ( $>3$ times more than glucose), its oxidation can produce a substantial amount of energy for the cell. In addition, fatty acid concentration significantly rises in some pathological situations (see next paragraph) in which they would become a privileged energy substrate. The plasma concentration of ketone bodies is even lower ( $\sim 10-100 \mu \mathrm{M}$ in the feeding adult rat) (Veech et al., 2001), but they can be produced locally from fatty acids by astrocytes themselves (Guzman and Blazquez, 2001). Our findings that CNTF directly acts on astrocytes to modify their metabolic profile in vivo and in vitro is new and suggests that CNTF may be a major regulator of peripheral and central cellular energy metabolism.

\section{Functional consequences of CNTF-induced brain metabolic plasticity}

Prolonged exposure to palmitate activates cell death pathways (Ulloth et al., 2003) and leads to ceramide synthesis in astrocytes (Blazquez et al., 2000). We found that CNTF-activated astrocytes were totally resistant to toxic palmitate levels in vitro, even when CNTF was removed from the medium during the $24 \mathrm{~h}$ exposure to palmitate. This result indicates that CNTF does not protect astrocytes through a delayed anti-cell death action but rather by a preexisting remodeling of the metabolic status of astrocytes. We also found that blockade of $\beta$-oxidation abolishes the protective effect of CNTF. Because palmitate $\beta$-oxidation is performed twice as efficiently in CNTF-activated astrocytes, our result suggests that the increased oxidation of fatty acids by CNTFactivated astrocytes prevents their accumulation and reduces their availability for ceramide neosynthesis (Fig. 11). This is totally consistent with the very recent observation that CNTF attenuates ceramide accumulation inside myocytes after lipid infusion (Watt et al., 2006a). Our results are of importance because a massive release of free fatty acids from phospholipids in the plasma membrane occurs in acute pathological conditions such as brain trauma and ischemia or a chronic neurodegenerative disease such as Alzheimer's disease (Phillis and O'Regan, 2004). All of these conditions also involve metabolic defects (Beal, 2000) and induce activation of astrocytes. In this regard, astrocyte activation appears to be a very efficient adaptive response to face a shortage in energetic supply and accumulation of toxic metabolites at the same time.

We also provided evidence that CNTF-activated astrocytes were highly resistant to glycolytic inhibition and that this effect was partially mediated by the transport of monocarboxylates. It was not possible to determine which of the monocarboxylates (ketone bodies, lactate, or pyruvate) was involved in CNTFprotective effects. However, the production of pyruvate and lactate is dependent on the glycolytic flux, and their concentrations may be lowered by IAA exposure. On the contrary, ketone bodies are totally independent from glycolysis, and their rate of oxidation was significantly increased with CNTF, making them more suitable candidates. 


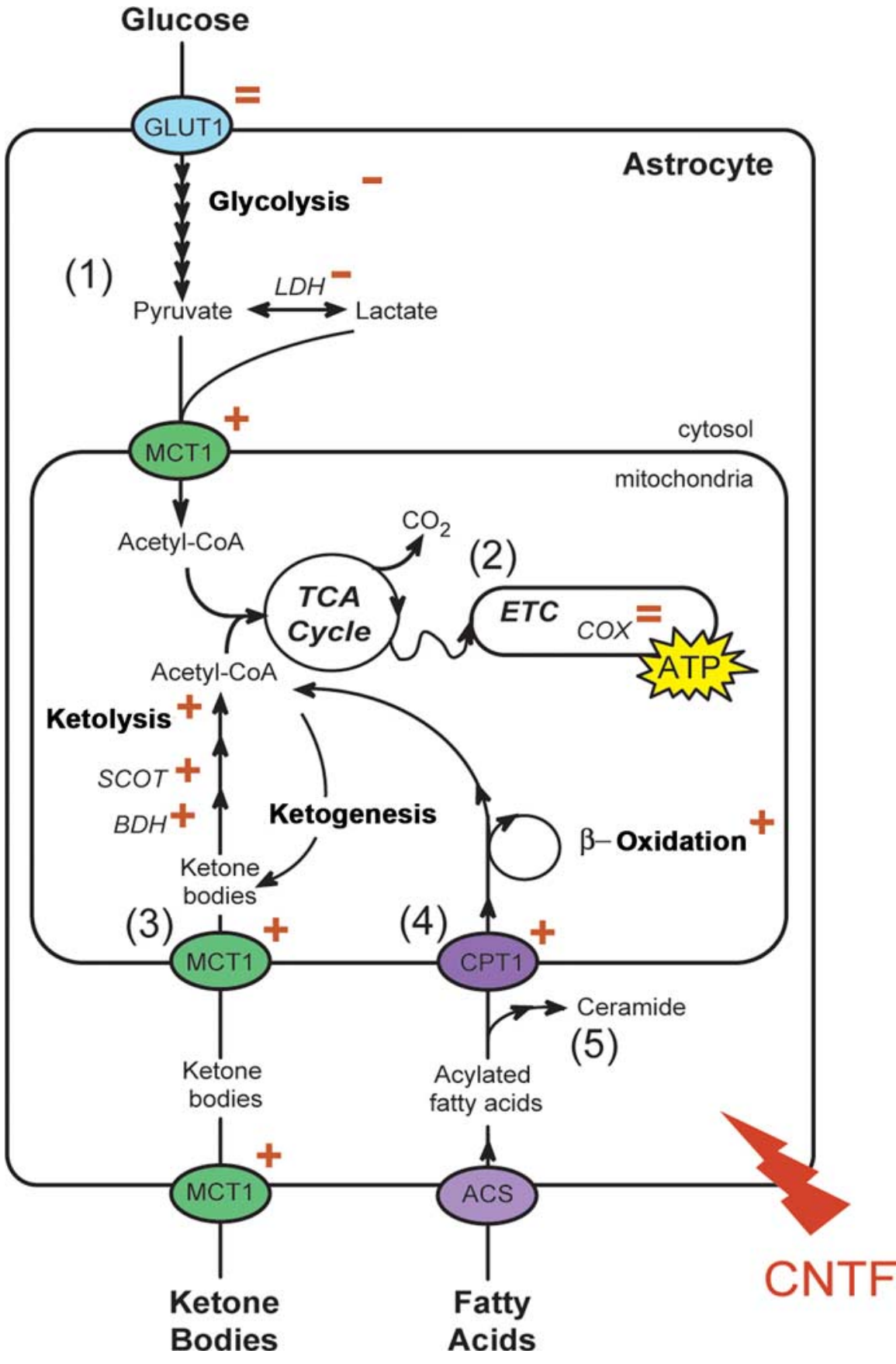

Figure 11. The pathways for energy production in astrocytes: effects of CNTF. (1) Glucose is taken up by astrocytes through the transporter Glut- 1 and is oxidized into pyruvate through glycolysis. LDH transforms pyruvate into lactate that may be exported to the neurons. (2) Pyruvate and lactate enter the mitochondria where they are further oxidized through the TCA cycle and the electron transfer chain (ETC) to produce ATP. (3) Ketone bodies can also fuel the TCA cycle with acetyl-CoA after cellular and mitochondrial uptake by MCT1 and oxidation by BDH and SCOT. (4) Fatty acids are exclusively metabolized by astrocytes through acylation by acyl-CoA synthase (ACS), uptake by CPT1 and $\beta$-oxidation to produce acetyl-COA that may either fuel the TCA cycle or be condensed into ketone bodies through ketogenesis. Ketone bodies, as lactate, may be given to neurons. (5) The fatty acid palmitate, once acylated, may alternatively fuel the pathway of ceramide neosynthesis, which is associated with apoptotic cell death. CNTF decreases glucose uptake and LDH activity. The expression of several key enzymes/transporters of ketolysis and $\beta$-oxidation are enhanced, consistent with the observed increase in the rate of ketone bodies and fatty acid oxidation. CNTF metabolic targets are represented with plus, equal, and minus red signs. Enzymes appear in italic. mitochondrial electron transport chain, bypassing glycolysis and PDH. Indeed, ketone bodies protect brain cells against situations of energy impairment, such as glucose deprivation, glycolytic inhibition on hippocampal slices from young rats (Izumi et al., 1998), ischemia (Marie et al., 1987), and blockade of mitochondrial complex I by MPTP in mice (Tieu et al., 2003). More generally, ketolysis seems to be more efficient than glycolysis to fuel the TCA cycle because it is performed in three rapid reactions within the mitochondria, whereas glycolysis requires 10 cytoplasmic reactions and mitochondrial uptake of $\mathrm{NADH}, \mathrm{H}^{+}$, pyruvate, and lactate (Veech et al., 2001). Most importantly, glycolysis requires ATP investment, which is problematic in conditions of energy depletion.

\section{CNTF-activated astrocytes as neuroprotective agents}

The activation of astrocytes is thought to induce neuronal demise in diverse pathological situations through release of toxic molecules, reversal of glutamate transport, or inhibition of axonal regrowth (Kalman, 2004). Our results, however, indicate that CNTF-activated astrocytes acquire an improved capacity to deal with metabolic impairment that leads to an increased neuronal survival. We have demonstrated previously that CNTF activation of astrocytes improved handling of toxic levels of extracellular glutamate (Escartin et al., 2006). Excitotoxicity is also involved in several pathological situations and is even exacerbated by metabolic dysfunction. The fact that CNTF-activated astrocytes are more efficient to cope with both insults may underlie the important neuroprotective effects of CNTF in various in vivo models of excitotoxic cell death (Anderson et al., 1996; de Almeida et al., 2001), ischemia (Hermann et al., 2001), and chronic metabolic impairment (Mittoux et al., 2000, 2002). CNTF has been tested clinically for the treatment of amyotrophic lateral sclerosis (Aebischer et al., 1996) and Huntington's disease (Bachoud-Levi et al., 2000), in which glutamate toxicity and metabolic deficiency play a key role (Beal, 1992). Considering the beneficial effects observed in the present study, our results strongly support the concept of testing CNTF as a therapeutic candidate for these incurable diseases.
The ability to efficiently use alternative substrates besides glucose may improve the resistance to a shortage in glucose supply, or inhibition of glycolysis/pyruvate dehydrogenase (PDH) complex. Oxidation of ketone bodies and fatty acids produces acetyl-CoA, $\mathrm{FADH}_{2}$, and $\mathrm{NADH}, \mathrm{H}^{+}$that can directly fuel the TCA cycle and the

\section{References}

Aebischer P, Schluep M, Deglon N, Joseph JM, Hirt L, Heyd B, Goddard M, Hammang JP, Zurn AD, Kato AC, Regli F, Baetge EE (1996) Intrathecal delivery of CNTF using encapsulated genetically modified xenogeneic cells in amyotrophic lateral sclerosis patients. Nat Med 2:696-699. 
Al-Ali SY, Robinson N (1982) Ultrastructural study of enzymes in reactive astrocytes: clarification of astrocytic activity. Histochem J 14:311-321.

Anderson KD, Panayotatos N, Corcoran TL, Lindsay RM, Wiegand SJ (1996) Ciliary neurotrophic factor protects striatal output neurons in an animal model of Huntington disease. Proc Natl Acad Sci USA 93:7346-7351.

Auestad N, Korsak RA, Morrow JW, Edmond J (1991) Fatty acid oxidation and ketogenesis by astrocytes in primary culture. J Neurochem 56:1376-1386.

Bachoud-Levi AC, Deglon N, Nguyen JP, Bloch J, Bourdet C, Winkel L, Remy P, Goddard M, Lefaucheur JP, Brugieres P, Baudic S, Cesaro P, Peschanski M, Aebischer P (2000) Neuroprotective gene therapy for Huntington's disease using a polymer encapsulated BHK cell line engineered to secrete human CNTF. Hum Gene Ther 11:1723-1729.

Bazan Jr NG (1970) Effects of ischemia and electroconvulsive shock on free fatty acid pool in the brain. Biochim Biophys Acta 218:1-10.

Bazan Jr NG (1971) Changes in free fatty acids of brain by drug-induced convulsions, electroshock and anaesthesia. J Neurochem 18:1379-1385.

Beal MF (1992) Does impairment of energy metabolism result in excitotoxic neuronal death in neurodegenerative illnesses? Ann Neurol 31:119-130.

Beal MF (2000) Energetics in the of neurodegenerative diseases. Trends Neurosci 23:298-304.

Bilger A, Nehlig A (1992) Quantitative histochemical changes in enzymes involved in energy metabolism in the rat brain during postnatal development. II. Glucose-6-phosphate dehydrogenase and beta-hydroxybutyrate dehydrogenase. Int J Dev Neurosci 10:143-152.

Blazquez C, Sanchez C, Velasco G, Guzman M (1998) Role of carnitine palmitoyltransferase $\mathrm{I}$ in the control of ketogenesis in primary cultures of rat astrocytes. J Neurochem 71:1597-1606.

Blazquez C, Woods A, de Ceballos ML, Carling D, Guzman M (1999) The AMP-activated protein kinase is involved in the regulation of ketone body production by astrocytes. J Neurochem 73:1674-1682.

Blazquez C, Galve-Roperh I, Guzman M (2000) De novo-synthesized ceramide signals apoptosis in astrocytes via extracellular signal-regulated kinase. FASEB J 14:2315-2322.

de Almeida LP, Zala D, Aebischer P, Deglon N (2001) Neuroprotective effect of a CNTF-expressing lentiviral vector in the quinolinic acid rat model of Huntington's disease. Neurobiol Dis 8:433-446.

Dufour F, Koning E, Nehlig A (2003) Basal levels of metabolic activity are elevated in Genetic Absence Epilepsy Rats from Strasbourg (GAERS): measurement of regional activity of cytochrome oxidase and lactate dehydrogenase by histochemistry. Exp Neurol 182:346-352.

Ebert D, Haller RG, Walton ME (2003) Energy contribution of octanoate to intact rat brain metabolism measured by ${ }^{13} \mathrm{C}$ nuclear magnetic resonance spectroscopy. J Neurosci 23:5928-5935.

Edmond J (1992) Energy metabolism in developing brain cells. Can J Physiol Pharmacol 70:S118-S129.

Edmond J, Robbins RA, Bergstrom JD, Cole RA, de Vellis J (1987) Capacity for substrate utilization in oxidative metabolism by neurons, astrocytes, and oligodendrocytes from developing brain in primary culture. J Neurosci Res 18:551-561.

Escartin C, Brouillet E, Gubellini P, Trioulier Y, Jacquard C, Smadja C, Knott GW, Kerkerian-Le Goff L, Deglon N, Hantraye P, Bonvento G (2006) Ciliary neurotrophic factor activates astrocytes, redistributes their glutamate transporters GLAST and GLT-1 to raft microdomains, and improves glutamate handling in vivo. J Neurosci 26:5978-5989.

Gloaguen I, Costa P, Demartis A, Lazzaro D, Di Marco A, Graziani R, Paonessa G, Chen F, Rosenblum CI, Van der Ploeg LH, Cortese R, Ciliberto G, Laufer R (1997) Ciliary neurotrophic factor corrects obesity and diabetes associated with leptin deficiency and resistance. Proc Natl Acad Sci USA 94:6456-6461.

Greene JG, Greenamyre JT (1995) Characterization of the excitotoxic potential of the reversible succinate dehydrogenase inhibitor malonate. J Neurochem 64:430-436.

Guzman M, Blazquez C (2001) Is there an astrocyte-neuron ketone body shuttle? Trends Endocrinol Metab 12:169-173.

Hardie DG, Hawley SA, Scott JW (2006) AMP-activated protein kinasedevelopment of the energy sensor concept. J Physiol (Lond) 574:7-15.

Herard AS, Dubois A, Escartin C, Tanaka K, Delzescaux T, Hantraye P, Bonvento $G$ (2005) Decreased metabolic response to visual stimulation in the superior colliculus of mice lacking the glial glutamate transporter GLT-1. Eur J Neurosci 22:1807-1811.

Hermann DM, Kilic E, Kugler S, Isenmann S, Bahr M (2001) Adenovirusmediated GDNF and CNTF pretreatment protects against striatal injury following transient middle cerebral artery occlusion in mice. Neurobiol Dis 8:655-666.

Izumi Y, Ishii K, Katsuki H, Benz AM, Zorumski CF (1998) beta-Hydroxybutyrate fuels synaptic function during development. Histological and physiological evidence in rat hippocampal slices. J Clin Invest 101:1121-1132.

Kacem K, Lacombe P, Seylaz J, Bonvento G (1998) Structural organization of the perivascular astrocyte endfeet and their relationship with the endothelial glucose transporter: a confocal microscopy study. Glia 23:1-10.

Kalman M (2004) Glial reaction and reactive glia. In: Non neuronal cells of the nervous system: function and dysfunction (Hertz L, ed), pp 787-835 Amsterdam: Elsevier.

Lambert PD, Anderson KD, Sleeman MW, Wong V, Tan J, Hijarunguru A, Corcoran TL, Murray JD, Thabet KE, Yancopoulos GD, Wiegand SJ (2001) Ciliary neurotrophic factor activates leptin-like pathways and reduces body fat, without cachexia or rebound weight gain, even in leptinresistant obesity. Proc Natl Acad Sci USA 98:4652-4657.

Lipton P (1999) Ischemic cell death in brain neurons. Physiol Rev 79:1431-1568.

Marie C, Bralet AM, Bralet J (1987) Protective action of 1,3-butanediol in cerebral ischemia. A neurologic, histologic, and metabolic study. J Cereb Blood Flow Metab 7:794-800.

Mittoux V, Joseph JM, Conde F, Palfi S, Dautry C, Poyot T, Bloch J, Deglon N, Ouary S, Nimchinsky EA, Brouillet E, Hof PR, Peschanski M, Aebischer P, Hantraye P (2000) Restoration of cognitive and motor functions by ciliary neurotrophic factor in a primate model of Huntington's disease. Hum Gene Ther 11:1177-1187.

Mittoux V, Ouary S, Monville C, Lisovoski F, Poyot T, Conde F, Escartin C, Robichon R, Brouillet E, Peschanski M, Hantraye P (2002) Corticostriatopallidal neuroprotection by adenovirus-mediated ciliary neurotrophic factor gene transfer in a rat model of progressive striatal degeneration. J Neurosci 22:4478-4486.

Pellerin L, Magistretti PJ (1994) Glutamate uptake into astrocytes stimulates aerobic glycolysis: a mechanism coupling neuronal activity to glucose utilization. Proc Natl Acad Sci USA 91:10625-10629.

Phillis JW, O’Regan MH (2004) A potentially critical role of phospholipases in central nervous system ischemic, traumatic, and neurodegenerative disorders. Brain Res Brain Res Rev 44:13-47.

Pierre K, Pellerin L, Debernardi R, Riederer BM, Magistretti PJ (2000) Cellspecific localization of monocarboxylate transporters, MCT1 and MCT2, in the adult mouse brain revealed by double immunohistochemical labeling and confocal microscopy. Neuroscience 100:617-627.

Sleeman MW, Garcia K, Liu R, Murray JD, Malinova L, Moncrieffe M, Yancopoulos GD, Wiegand SJ (2003) Ciliary neurotrophic factor improves diabetic parameters and hepatic steatosis and increases basal metabolic rate in db/db mice. Proc Natl Acad Sci USA 100:14297-14302.

Smith B (1963) Dehydrogenase activity in reactive and neoplastic astrocytes. Brain 86:89-94.

Sokoloff L (1973) Metabolism of ketone bodies by the brain. Annu Rev Med 24:271-280.

Tieu K, Perier C, Caspersen C, Teismann P, Wu DC, Yan SD, Naini A, Vila M, Jackson-Lewis V, Ramasamy R, Przedborski S (2003) D-betahydroxybutyrate rescues mitochondrial respiration and mitigates features of Parkinson disease. J Clin Invest 112:892-901.

Ulloth JE, Casiano CA, De Leon M (2003) Palmitic and stearic fatty acids induce caspase-dependent and -independent cell death in nerve growth factor differentiated PC12 cells. J Neurochem 84:655-668.

Veech RL, Chance B, Kashiwaya Y, Lardy HA, Cahill Jr GF (2001) Ketone bodies, potential therapeutic uses. IUBMB Life 51:241-247.

Voutsinos-Porche B, Bonvento G, Tanaka K, Steiner P, Welker E, Chatton JY, Magistretti PJ, Pellerin L (2003) Glial glutamate transporters mediate a functional metabolic crosstalk between neurons and astrocytes in the mouse developing cortex. Neuron 37:275-286.

Watt MJ, Hevener A, Lancaster GI, Febbraio MA (2006a) Ciliary neurotrophic factor prevents acute lipid-induced insulin resistance by attenuating ceramide accumulation and phosphorylation of c-Jun N-terminal kinase in peripheral tissues. Endocrinology 147:2077-2085.

Watt MJ, Dzamko N, Thomas WG, Rose-John S, Ernst M, Carling D, Kemp BE, Febbraio MA, Steinberg GR (2006b) CNTF reverses obesity-induced insulin resistance by activating skeletal muscle AMPK. Nat Med 12:541-548.

Xu B, Dube MG, Kalra PS, Farmerie WG, Kaibara A, Moldawer LL, Martin D, Kalra SP (1998) Anorectic effects of the cytokine, ciliary neurotropic factor, are mediated by hypothalamic neuropeptide Y: comparison with leptin. Endocrinology 139:466-473. 